\title{
Lymph nodes regression grade is a predictive marker for rectal cancer after neoadjuvant therapy and radical surgery
}

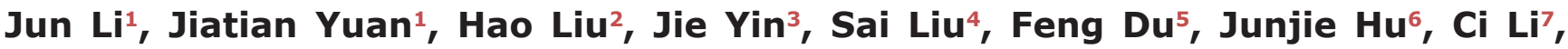 \\ Xiangke Niu ${ }^{8}$, Bo Lv' ${ }^{1}$, Shasha Xing ${ }^{9}$ \\ ${ }^{1}$ General Surgery Department, Affiliated Hospital/Clinical Medical College of Chengdu University, Chengdu, P.R. China \\ ${ }^{2}$ General Surgery Department, Second Affiliated Hospital of Jilin University, Changchun, P.R. China \\ ${ }^{3}$ General Surgery Department, Xuzhou Central Hospital, Xuzhou, P.R. China \\ ${ }^{4}$ Surgical Department of Gastrointestinal Diseases, Beijing Youan Hospital of Capital Medical University, Beijing, P.R. China \\ ${ }^{5}$ Internal Medicine-Oncology, Cancer Institute/Hospital, Peking Union Medical College and Chinese Academy of Medical \\ Sciences, Beijing, P.R. China \\ ${ }^{6}$ Gastrointestinal Tumor Surgery, Hubei Cancer Hospital, Wuhan, P.R. China \\ ${ }^{7}$ Department of Pathology, Affiliated Hospital/Clinical Medical College of Chengdu University, Chengdu, P.R. China \\ ${ }^{8}$ Department of Radiology, Affiliated Hospital/Clinical Medical College of Chengdu University, Chengdu, P.R. China \\ ${ }^{9}$ Central Lab, Affiliated Hospital/Clinical Medical College of Chengdu University, Chengdu, P.R. China \\ Correspondence to: Jun Li, e-mail: junl_paper@sina.com \\ Jiatian Yuan, e-mail: jiatianyuan_pro@sina.com \\ Shasha Xing, e-mail: xingshasha1230@126.com
}

Keywords: lymph node, regression grading, rectal cancer, neoadjuvant therapy

Received: October 10, $2015 \quad$ Accepted: February 08, 2016

Published: February 25, 2016

\section{ABSTRACT}

\begin{abstract}
Neoadjuvant therapy (NT) for rectal cancer (RC) reduces primary tumors and involved lymph nodes. While a prognostic value of tumor regression grade (TRG) has been identified, involved lymph node regression grade (LRG) has not been systematically evaluated. Here, we evaluated the association of LRG with oncologic outcomes of RC patients after NT followed by radical surgery. 347 patients with locally advanced RC who received NT and then underwent radical surgery were retrospectively recruited between 2004 and 2011. Response to NT was evaluated by a 3-tier LRG and TRG based on the ratio of residual tumor to fibrosis. LRG was assessed in all patients (LRG 0, 170 patients [49.0\%]; LRG 1, 100 patients [28.8\%]; and LRG 2, 77 patients [22.2\%]). LRG correlated with 5-year distant metastasis and 5-year disease free survival ( $p=0.029$ and 0.023 , respectively). LRG also correlated with TRG $(p=0.017)$. We conclude that the LRG system may be an independent predictive factor of long-term oncologic outcomes of rectal cancer patients after NT and radical surgery.
\end{abstract}

\section{INTRODUCTION}

Neoadjuvant therapy (NT) in rectal cancer (RC) downstages primary tumors and reduces local recurrence in locally advanced rectal cancer $[1,2]$. Large numbers of NT trials have explored the use of tumor regression grading (TRG) as a primary end-point. Various grading systems, including the Manard, Dowrak, Dowark/ Rodel, AJCC and MSKCC have been proposed. All of these use the percentage of tumor cells relative to fibrosis. However, TRG scores do not account for the involvement of lymph nodes, which is an important prognostic parameter [3]. In this regard, Perez et al. [4] reported that histologic regression could be observed in mesorectal lymph nodes after NT. Furthermore, studies indicated that TRG of primary tumors may predict lymph node responses [5, 6]. While Caricato et al. [7] demonstrated that LRG correlated with TRG in primary tumors, but they did not examine the impact of LRG on oncologic outcomes. We therefore evaluated the impact of LRG on oncologic outcomes including local recurrence (LR), distant metastasis (DM), and 5-year disease-free survival (DFS), and the LRG correlation with TRG in primary RC tumors. 


\section{RESULTS}

\section{Patient characteristics and association of LRG with clinicopathologic factors}

347 patients with locally advanced rectal cancer who received radical surgery in 6-8weeks after NT were identified in this retrospective study. A complete pathologic regression (pCR, ypT0N0) was seen in 46 patients (13.3\%). In sum, 4012 lymph nodes were detected in all patients; the mean number of LNs was $11.6 \pm 2.3$ (range: $1-44$ nodes). Of note, $66(19.0 \%)$ patients had the number of LNs less than 10. In total, 676 metastasis mesorectal lymph nodes $(16.8 \%)$ were found; the mean numbers of positive LNs were $2.1 \pm 0.3$ and $6.3 \pm 1.1$ in ypN1-2, respectively. LRG was assessed in all patients (LRG 0, 170 patients [49.0\%]; LRG 1, 100 patients [28.8\%]; LRG 2, 77 patients [22.2\%]). cT stage and $\mathrm{cN}$ stage did not predict LRG $(P=0.815$ and 0.432 , respectively) (Table 1 ).

The association of LRG with histopathologic factors is recorded in Table 2. Radical resection of the primary tumor (R0) was performed in all patients. LRG correlated with TRG score, ypT stage, ypN stage, and venous invasion $(P<0.05)$. No significant association was found between LRG and tumor differentiation degree, lymphatic invasion, and tumor deposits after radical surgery.

\section{LRG as a prognostic factor for DFS}

The 5-year DFS rate for 347 patients after radical surgery was $80.4 \%$. 34 patients had local recurrence, and 34 patients had distant metastasis. None of the patients suffered from both local recurrence and distant metastasis. $148(85.9 \%), 78(78.0 \%)$ and $55(71.4 \%)$ patients with LRG 0,1 , and 2 experienced a 5-year DFS, respectively. TRG correlated with 5 -year distant metastasis $(P=0.035)$, but failed to correlate with both 5-year local recurrence and 5-year DFS ( $P=0.531$ and 0.576 , respectively). LRG correlated with 5-year distant metastasis and 5-year DFS ( $P=0.029$ and 0.023 , respectively). Disease free survival curve for LRG scores is shown in Figure 1. As listed in Table 3, other factors that correlated with DFS by univariable analysis included the ypT and ypN stage, lymphatic invasion and venous invasion (all $P<0.05$ ). Using multivariable analysis, the results indicated that two variables including ypT and ypN were independent risk factors for three end-points. LRG was a significant independent predictor of 5-year distant metastasis and 5 -year DFS but not for 5-year local recurrence (Table 4).

\section{Relationship between TRG and primary tumor}

All cases were enrolled into the model of correlation test. Spearman correlation test was 3.22 and $p$ value was statistically significant $(P=0.017) . \mathrm{LRG}$ correlated with TRG.
In 198 patients with ypN0, we examined the preoperative CT and MRI images and found that 31 patients had LN+ lesions. Based on this finding, we assumed that NT killed all the tumor cells but pathologists could not find the residual lymph nodes; this group of patients should have good tumor regression in lymph nodes. Thus, these 31 cases $(15.6 \%)$ were considered as LRG 0 . We found that patients with fibrosis (139 cases) and without fibrosis (31 cases) had similar 5-year DFS $(83.9 \%$ vs. $86.3 \%, P=0.080)$ (Table 5 ).

\section{DISCUSSION}

Salzer-Kuntschik et al [8] first described tumor regression grade in osteosarcoma after chemotherapy in 1983. In 2002, Bouzorene et al. [9] retrospectively reviewed the resection specimens from 102 patients with locally advanced rectal cancer after preoperative radiotherapy and indicated that tumor regression was a predictive factor for survival. Mandard et al. [10] defined tumor regression in five grades based on residual tumor and fibrosis. In recent years, the TRG grading systems, including the Manard, Dowrak, Dowark/Rodel, AJCC and MSKCC systems, have been proposed. These systems are all based on the percentage of tumor cells relative to fibrosis. However, TRG scores do not account for the involvement of lymph nodes, which are an important parameter of prognosis. In 2007, Caricato et al. [7] reported mesorectal LRG in rectal cancer after NT, but they lacked the long-term data needed to analyze DFS. As far as we know, our study is the first to evaluate the association of LRG with long-term oncologic outcomes.

The results of this study indicate that LRG can predict distant metastasis and DFS, and that LRG is an independent prognostic factor for RC after NT and radical surgery for both DM and DFS. Compared with TRG, we found that LRG correlated with 5-year distant metastasis. Based on the CAO/ARO/AIO-94 trial, Rodel et al. [11] concluded that TRG may be a predictive factor for 5 -year distant metastasis. The study group then updated the results and concluded that TRG was a significant prognostic factor for 10-year distant metastasis and DFS [12]. However, our results indicate that TRG may not correlate with 5-year DFS. These differences may be caused by different treatment regimens, including radiation dose, medication used in chemotherapy and pathology practices, as well as differences in duration between NT and surgery. In fact, Kalady et al. [13] reported that patients with an incomplete response at 6 weeks might become pCR at 12 weeks. Accordingly, the interval time between NT and radical surgery was a predominant influence in a pCR, which may impact TRG classification and interfere with the result of the trials. Thus, we consider that TRG alone is not a reliable prognostic factor.

Our study shows that LRG correlates with TRG, as reported previously [5-7]. This indicates that the 
Table 1: Association of LRG with pretreatment and tumor characteristics in 347 patients

\begin{tabular}{|c|c|c|c|c|c|c|c|c|c|}
\hline \multirow[t]{2}{*}{ Variable } & \multicolumn{2}{|c|}{ LRG 0} & \multicolumn{2}{|c|}{ LRG 1} & \multicolumn{2}{|c|}{ LRG 2} & \multirow{2}{*}{$\begin{array}{c}\text { Total } \\
\text { No. }\end{array}$} & \multirow[t]{2}{*}{$X^{2}$} & \multirow[t]{2}{*}{$p$} \\
\hline & No. & $\%$ & No. & $\%$ & No. & $\%$ & & & \\
\hline Overall & 170 & 49.0 & 100 & 28.8 & 77 & 22.2 & 347 & & \\
\hline
\end{tabular}

Age, years

\begin{tabular}{|c|c|c|c|c|c|c|c|c|c|}
\hline$\leq 60$ & 88 & 46.3 & 58 & 30.5 & 44 & 23.2 & 190 & 1.216 & 0.544 \\
\hline$>60$ & 82 & 52.2 & 42 & 26.8 & 33 & 21.0 & 157 & & \\
\hline
\end{tabular}

Gender

$\begin{array}{llllllllll}\text { Male } & 105 & 50.7 & 62 & 30.0 & 40 & 19.3 & 207 & 2.443 & 0.295 \\ \text { Female } & 65 & 46.4 & 38 & 27.1 & 37 & 26.4 & 140 & \end{array}$

Distance from anal verge, $\mathrm{cm}$

$\begin{array}{llllllllll}\leq 5 & 93 & 49.2 & 55 & 29.1 & 41 & 21.7 & 189 & 0.062 & 0.97 \\ >5 & 77 & 48.7 & 45 & 28.5 & 36 & 22.8 & 158 & \end{array}$

Preoperative CEA

$\begin{array}{lllllllll}\text { negative } & 90 & 47.4 & 54 & 28.4 & 46 & 24.2 & 190 & 1.261 \\ \text { positive } & 70 & 51.5 & 40 & 29.4 & 26 & 19.1 & 136 & \\ \text { unknown } & 10 & 47.6 & 6 & 28.6 & 5 & 23.8 & 21 & \end{array}$

Preoperative treatment

\begin{tabular}{|c|c|c|c|c|c|c|c|c|c|}
\hline $\begin{array}{l}\text { Preoperative } \\
\text { CRT }\end{array}$ & 104 & 48.8 & 61 & 28.6 & 48 & 22.5 & 213 & 0.039 & 0.981 \\
\hline $\begin{array}{l}\text { Radiotherapy } \\
\text { only }\end{array}$ & 66 & 49.3 & 39 & 29.1 & 29 & 21.6 & 134 & & \\
\hline \multicolumn{10}{|l|}{ T stage } \\
\hline cT2 & 70 & 50.7 & 39 & 28.3 & 29 & 21.0 & 138 & 2.948 & 0.815 \\
\hline cT3 & 58 & 47.5 & 34 & 27.9 & 30 & 24.6 & 122 & & \\
\hline cT4 & 41 & 48.8 & 25 & 29.8 & 18 & 21.4 & 84 & & \\
\hline unknown & 1 & 33.3 & 2 & 66.7 & 0 & 0.0 & 3 & & \\
\hline \multicolumn{10}{|l|}{ N stage } \\
\hline cNO & 90 & 51.4 & 45 & 25.7 & 40 & 22.9 & 175 & 1.679 & 0.432 \\
\hline $\mathrm{cN}+$ & 80 & 46.5 & 55 & 32.0 & 37 & 21.5 & 172 & & \\
\hline
\end{tabular}


Table 2: Association of LRG with pathological factors after NT and radical surgery

\begin{tabular}{|c|c|c|c|c|c|c|c|c|c|}
\hline \multirow[t]{2}{*}{ Variable } & \multicolumn{2}{|c|}{ LRG 0} & \multicolumn{2}{|c|}{ LRG 1} & \multicolumn{2}{|c|}{ LRG 2} & \multirow[t]{2}{*}{ Total } & \multirow[t]{2}{*}{$X^{2}$} & \multirow[t]{2}{*}{$P$} \\
\hline & No. & $\%$ & No. & $\%$ & No. & $\%$ & & & \\
\hline Overall & 170 & 49.0 & 100 & 28.8 & 77 & 22.2 & 347 & & \\
\hline \multicolumn{10}{|l|}{ TRG score } \\
\hline 0 & 46 & 66.7 & 23 & 33.3 & 0 & 0.0 & 69 & 37.733 & $<0.0001$ \\
\hline 1 & 65 & 48.9 & 39 & 29.3 & 29 & 21.8 & 133 & & \\
\hline 2 & 59 & 38.1 & 38 & 24.5 & 58 & 37.4 & 155 & & \\
\hline \multicolumn{10}{|l|}{ ypT stage } \\
\hline урт0 & 33 & 47.8 & 21 & 30.4 & 15 & 21.7 & 69 & 31.178 & $<0.0001$ \\
\hline урT1 & 60 & 49.6 & 40 & 33.1 & 21 & 17.4 & 121 & & \\
\hline урТ2 & 55 & 57.9 & 20 & 21.1 & 20 & 21.1 & 95 & & \\
\hline урT3 & 16 & 50.0 & 14 & 43.8 & 2 & 16.2 & 32 & & \\
\hline урт4 & 6 & 20.0 & 5 & 16.7 & 19 & 63.3 & 30 & & \\
\hline \multicolumn{10}{|l|}{ ypN stage } \\
\hline ypN0 & 155 & 78.3 & 0 & 0.0 & 43 & 21.7 & 198 & 68.109 & $<0.0001$ \\
\hline ypN1 & 9 & 5.9 & 82 & 82.0 & 11 & 10.8 & 102 & & \\
\hline ypN2 & 6 & 12.8 & 18 & 18.0 & 23 & 48.9 & 47 & & \\
\hline \multicolumn{10}{|c|}{ Tumor differentiation degree } \\
\hline poor & 39 & 48.8 & 23 & 28.8 & 18 & 22.5 & 80 & 0.033 & 0.997 \\
\hline moderate & 59 & 49.2 & 35 & 29.2 & 26 & 21.7 & 120 & & \\
\hline well & 72 & 49.0 & 42 & 28.6 & 33 & 22.5 & 147 & & \\
\hline \multicolumn{10}{|c|}{ Lymphatic invasion } \\
\hline negative & 134 & 48.9 & 79 & 28.8 & 61 & 22.3 & 274 & 0.005 & 0.997 \\
\hline positive & 36 & 49.3 & 21 & 28.8 & 16 & 21.9 & 73 & & \\
\hline \multicolumn{10}{|c|}{ Venous invasion } \\
\hline negative & 119 & 49.0 & 60 & 24.7 & 64 & 26.3 & 243 & 11.076 & 0.004 \\
\hline positive & 51 & 49.0 & 38.5 & 28.9 & 13 & 12.5 & 104 & & \\
\hline \multicolumn{10}{|c|}{ Tumor deposits } \\
\hline negative & 136 & 48.8 & 72 & 25.8 & 51 & 18.3 & 279 & 5.823 & 0.054 \\
\hline positive & 34 & 50.0 & 28 & 41.2 & 26 & 38.2 & 68 & & \\
\hline
\end{tabular}


primary tumor and positive lymph nodes respond similarly to neoadjuvant therapy, which suggests TRG is predictive of the incidence of involved lymph nodes after NT; hence, some authors suggested that TRG might be helpful in selecting patients suitable for a surgically conservative procedure such as local excision [5] or a wait-and-see policy [14-16]. However, in our previous study, we defined a new tumor regression grade (NTRG), which was calculated as the TRG score plus a lymph node score ( $\mathrm{pN}$ category) and we indicated that NTRG was superior to TRG alone to predict the longterm prognosis of rectal cancer after NT followed by radical surgery [17]. We calculated NTRG using $\mathrm{pN}$ stage score not suing LRG, considering that LRG was similar to TRG according to the results of other authors. Interesting, in the present study, we conclude that LRG strongly correlates with TRG, and more surprising, LRG may be useful to assess the long-term prognosis of RC patients. Although our results indicate that LRG may predict long-term oncologic outcomes, some questions remain. First, it is difficult to assess how many of the ypN0 patients with only microscopic LN involvement have really been downstaged. Second, the number of retrieved lymph nodes from patients after NT is lower than in patients treated with radical surgery only. This indicates that NT damages the structure of LNs so that we could not assess whether a small focus of fibrotic tissue found was a pre-treatment normal or metastatic LN. Lastly, pathologists could not distinguish patients with LNs without fibrosis and residual tumor in ypN0 into LRG 0 (complete response) or LRG 2 (no response). Given that we found that patients with and without fibrosis had similar oncologic outcomes, in this study, we enrolled 31 ypN0 patients into LRG 0 (Table 5). But still, we should recognize that complete response in patients with clinical LN+ by MRI but nothing on pathology is not a safe assumption. Besides, does no fibrosis in LN really mean there was any tumor cell

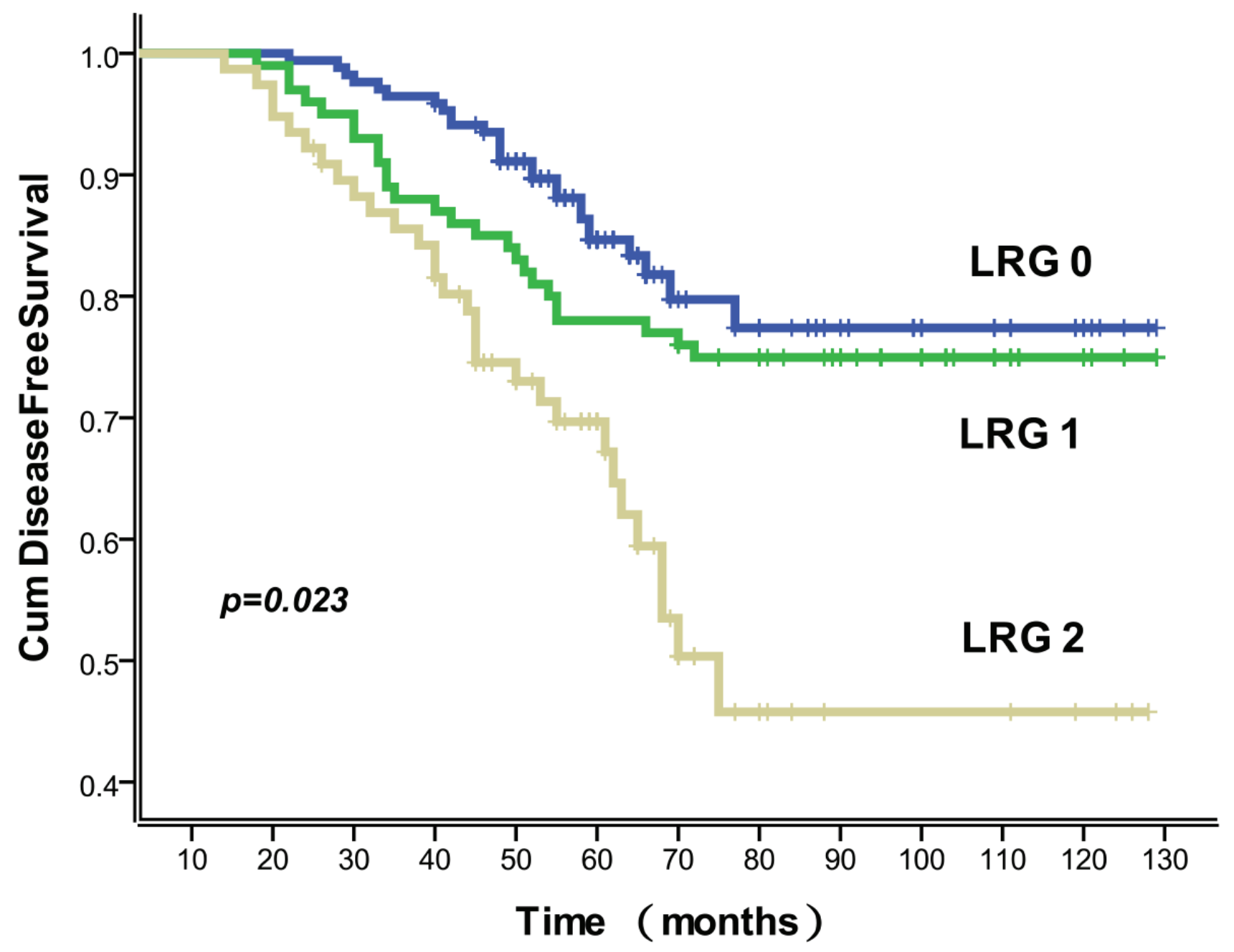

Figure 1: Association of LRG with disease free survival. Disease-free survival curves showed a significant relation to LRG. Data for all 347 cases were available. The 5-year disease-free survival rates for LRG 0-2 were 85.9\% (146/170), 78.0\% (78/100), and 71.4\% $(55 / 77)$, respectively. 
Table 3: Influence of different clinical and pathologic factors on 5-year prognosis after NT and radical surgery

\begin{tabular}{|c|c|c|c|c|c|c|c|c|c|}
\hline \multirow[t]{2}{*}{ Variables } & \multirow{2}{*}{$\begin{array}{c}\text { No. } \\
\text { of } \\
\text { Patients }\end{array}$} & \multicolumn{2}{|c|}{$\begin{array}{c}\text { Local } \\
\text { Recurrence }\end{array}$} & \multirow[t]{2}{*}{$P$} & \multicolumn{2}{|c|}{$\begin{array}{c}\text { Distant } \\
\text { Metastasis }\end{array}$} & \multirow[t]{2}{*}{$P$} & \multirow{2}{*}{$\begin{array}{c}\text { 5-Year } \\
\text { Disease Free } \\
\text { Survival }\end{array}$} & \multirow[t]{2}{*}{$p$} \\
\hline & & No. & $\%$ & & No. & $\%$ & & & \\
\hline Overall & 347 & 34 & 9.8 & & 34 & 9.8 & & $80.4 \%$ & \\
\hline \multicolumn{10}{|l|}{ Age, years } \\
\hline$\leq 60$ & 190 & 19 & 10.0 & 0.9 & 18 & 9.5 & 0.077 & $80.5 \%$ & 0.983 \\
\hline$>60$ & 157 & 15 & 9.6 & & 16 & 10.2 & & $80.3 \%$ & \\
\hline \multicolumn{10}{|l|}{ Gender } \\
\hline Male & 207 & 23 & 11.1 & 0.363 & 22 & 10.6 & 0.566 & $78.3 \%$ & 0.688 \\
\hline Female & 140 & 11 & 7.9 & & 12 & 8.6 & & $83.6 \%$ & \\
\hline \multicolumn{10}{|l|}{ ypT stage } \\
\hline урТ0 & 69 & 1 & 1.4 & 0.014 & 1 & 1.4 & 0.001 & $97.1 \%$ & 0.024 \\
\hline урT1 & 121 & 9 & 7.4 & & 9 & 7.4 & & $85.1 \%$ & \\
\hline урТ2 & 95 & 11 & 11.6 & & 8 & 8.4 & & $80.0 \%$ & \\
\hline урT3 & 32 & 6 & 18.8 & & 6 & 18.8 & & $62.5 \%$ & \\
\hline урТ4 & 30 & 7 & 23.3 & & 10 & 33.3 & & $43.3 \%$ & \\
\hline \multicolumn{10}{|l|}{ ypN stage } \\
\hline ypN0 & 198 & 9 & 4.5 & 0.001 & 15 & 7.6 & 0.044 & $87.9 \%$ & $<0.0001$ \\
\hline ypN1 & 102 & 13 & 12.7 & & 9 & 8.8 & & $78.4 \%$ & \\
\hline ypN2 & 47 & 12 & 25.5 & & 10 & 21.3 & & $53.2 \%$ & \\
\hline \multicolumn{10}{|c|}{ Tumor differentiation degree } \\
\hline poor & 80 & 13 & 16.3 & 0.089 & 13 & 16.3 & 0.083 & $67.5 \%$ & 0.468 \\
\hline moderate & 120 & 12 & 10.0 & & 11 & 9.2 & & $80.8 \%$ & \\
\hline well & 147 & 9 & 6.1 & & 9 & 6.1 & & $87.8 \%$ & \\
\hline \multicolumn{10}{|c|}{ Lymphatic invasion } \\
\hline negative & 274 & 13 & 4.7 & $<0.0001$ & 13 & 4.7 & $<0.0001$ & $90.5 \%$ & 0.001 \\
\hline positive & 73 & 21 & 28.8 & & 21 & 28.8 & & $42.5 \%$ & \\
\hline \multicolumn{10}{|c|}{ Venous invasion } \\
\hline negative & 243 & 9 & 3.7 & $<0.0001$ & 11 & 4.5 & $<0.0001$ & $91.8 \%$ & 0.005 \\
\hline positive & 104 & 25 & 24.0 & & 23 & 22.1 & & $53.8 \%$ & \\
\hline
\end{tabular}




\begin{tabular}{|c|c|c|c|c|c|c|c|c|c|}
\hline \multirow[t]{2}{*}{ Variables } & \multirow{2}{*}{$\begin{array}{c}\text { No. } \\
\text { of } \\
\text { Patients }\end{array}$} & \multicolumn{2}{|c|}{$\begin{array}{c}\text { Local } \\
\text { Recurrence }\end{array}$} & \multirow[t]{2}{*}{$P$} & \multicolumn{2}{|c|}{$\begin{array}{c}\text { Distant } \\
\text { Metastasis }\end{array}$} & \multirow[t]{2}{*}{$P$} & \multirow{2}{*}{$\begin{array}{c}\text { 5-Year } \\
\text { Disease Free } \\
\text { Survival }\end{array}$} & \multirow[t]{2}{*}{$p$} \\
\hline & & No. & $\%$ & & No. & $\%$ & & & \\
\hline \multicolumn{10}{|l|}{ Tumor deposits } \\
\hline negative & 279 & 26 & 9.3 & 0.584 & 25 & 9.0 & 0.341 & $88.9 \%$ & 0.406 \\
\hline positive & 68 & 8 & 11.8 & & 9 & 13.2 & & $75.0 \%$ & \\
\hline \multicolumn{10}{|c|}{ Postoperative chemotherapy } \\
\hline Yes & 285 & 22 & 7.7 & 0.014 & 25 & 8.8 & 0.219 & $83.5 \%$ & 0.287 \\
\hline No & 62 & 12 & 19.4 & & 9 & 14.5 & & $66.1 \%$ & \\
\hline \multicolumn{10}{|l|}{ TRG score } \\
\hline 0 (total) & 69 & 4 & 5.8 & 0.531 & 2 & 2.9 & 0.035 & $91.3 \%$ & 0.576 \\
\hline 1 (intermediate) & 133 & 13 & 9.8 & & 10 & 7.5 & & $82.7 \%$ & \\
\hline $\begin{array}{l}2 \text { (minor } \\
\text { and no) }\end{array}$ & 155 & 17 & 11.0 & & 22 & 14.2 & & $73.5 \%$ & \\
\hline \multicolumn{10}{|l|}{ LRG score } \\
\hline 0 (total) & 170 & 12 & 7.1 & 0.237 & 12 & 7.1 & 0.029 & $85.9 \%$ & 0.023 \\
\hline 1 (intermediate) & 100 & 12 & 12.0 & & 10 & 10.0 & & $78.0 \%$ & \\
\hline $\begin{array}{l}2 \text { (minor } \\
\text { and no) }\end{array}$ & 77 & 10 & 13.0 & & 12 & 15.6 & & $71.4 \%$ & \\
\hline
\end{tabular}

Table 4: Multivariate analysis for three end-points after NT and radical surgery

\begin{tabular}{|c|c|c|c|c|c|c|c|c|c|}
\hline \multirow[t]{2}{*}{ Variables } & \multicolumn{3}{|c|}{ 5-Year Local Recurrence } & \multicolumn{3}{|c|}{ 5-Year Distant Metastasis } & \multicolumn{3}{|c|}{ 5-Year Disease Free Survival } \\
\hline & HR & $95.0 \% \mathrm{CI}$ & $P$ & HR & $95.0 \% \mathrm{CI}$ & $\boldsymbol{P}$ & HR & $95.0 \% \mathrm{CI}$ & $P$ \\
\hline урТ & 0.47 & $\begin{array}{c}(0.29 \text { to } \\
0.80)\end{array}$ & 0.021 & 0.55 & $\begin{array}{c}(0.39 \text { to } \\
0.90)\end{array}$ & 0.040 & 0.54 & $\begin{array}{l}(0.31 \text { to } \\
0.71)\end{array}$ & 0.033 \\
\hline ypN & 2.37 & $\begin{array}{c}(1.74 \text { to } \\
2.95)\end{array}$ & 0.006 & 1.80 & $\begin{array}{c}(1.45 \text { to } \\
2.02)\end{array}$ & 0.039 & 2.58 & $\begin{array}{c}(2.25 \text { to } \\
3.05)\end{array}$ & $<0.0001$ \\
\hline $\begin{array}{l}\text { Lymphatic } \\
\text { invasion }\end{array}$ & 1.47 & $\begin{array}{c}(0.99 \text { to } \\
1.87)\end{array}$ & 0.083 & 1.13 & $\begin{array}{l}(0.90 \text { to } \\
1.28)\end{array}$ & 0.480 & 1.03 & $\begin{array}{l}(0.94 \text { to } \\
1.17)\end{array}$ & 0.086 \\
\hline Venous invasion & 1.12 & $\begin{array}{l}(0.69 \text { to } \\
1.47)\end{array}$ & 0.835 & 0.89 & $\begin{array}{l}(0.71 \text { to } \\
1.07)\end{array}$ & 0.713 & 1.20 & $\begin{array}{l}(0.99 \text { to } \\
1.47)\end{array}$ & 0.823 \\
\hline $\begin{array}{l}\text { Postoperative } \\
\text { chemotherapy }\end{array}$ & 1.24 & $\begin{array}{l}(0.93 \text { to } \\
1.43)\end{array}$ & 0.051 & - & & & - & & \\
\hline
\end{tabular}

(Continued) 


\begin{tabular}{|c|c|c|c|c|c|c|c|c|c|}
\hline \multirow[t]{2}{*}{ Variables } & \multicolumn{3}{|c|}{ 5-Year Local Recurrence } & \multicolumn{3}{|c|}{ 5-Year Distant Metastasis } & \multicolumn{3}{|c|}{ 5-Year Disease Free Survival } \\
\hline & HR & $95.0 \% \mathrm{CI}$ & $P$ & HR & $95.0 \% \mathrm{CI}$ & $P$ & HR & $95.0 \% \mathrm{CI}$ & $P$ \\
\hline TRG & - & & & 1.15 & $\begin{array}{c}(0.90 \text { to } \\
1.29)\end{array}$ & 0.198 & - & & \\
\hline LRG & - & & & 1.37 & $\begin{array}{c}\text { (1.11 to } \\
1.53)\end{array}$ & 0.032 & 1.68 & $\begin{array}{c}\text { (1.31 to } \\
2.59)\end{array}$ & 0.027 \\
\hline
\end{tabular}

Table 5: Association of ypN0 with oncologic outcomes in patients with and without fibrosis after NT and radical surgery

\begin{tabular}{|c|c|c|c|c|c|c|c|c|c|}
\hline \multirow[t]{2}{*}{ Variables } & \multirow{2}{*}{$\begin{array}{c}\text { No. } \\
\text { of } \\
\text { Patients }\end{array}$} & \multicolumn{2}{|c|}{$\begin{array}{l}\text { No. of Local } \\
\text { Recurrence }\end{array}$} & \multirow{2}{*}{$\begin{array}{c}\text { 5-Year Local } \\
\text { Recurrence } \\
\text { (p) }\end{array}$} & \multicolumn{2}{|c|}{$\begin{array}{l}\text { No. of Distant } \\
\text { Metastasis }\end{array}$} & \multirow{2}{*}{$\begin{array}{l}\text { 5-Year Distant } \\
\text { Metastasis } \\
(p)\end{array}$} & \multirow{2}{*}{$\begin{array}{c}\text { 5-Year } \\
\text { Disease Free } \\
\text { Survival }\end{array}$} & \multirow[t]{2}{*}{$p$} \\
\hline & & $\begin{array}{c}\text { All } \\
\text { Cases }\end{array}$ & $\begin{array}{l}\text { 5-Year } \\
\text { Cases }\end{array}$ & & $\begin{array}{c}\text { All } \\
\text { Cases }\end{array}$ & $\begin{array}{l}\text { 5-Year } \\
\text { Cases }\end{array}$ & & & \\
\hline LRG 0 & 170 & 13 & 12 & & 14 & 12 & & $85.9 \%$ & \\
\hline $\begin{array}{l}\text { ypN0 } \\
\text { without } \\
\text { fibrosis }\end{array}$ & 31 & 3 & 3 & 0.529 & 3 & 2 & 0.814 & $83.9 \%$ & 0.080 \\
\hline $\begin{array}{l}\text { ypN0 } \\
\text { with } \\
\text { fibrosis }\end{array}$ & 139 & 10 & 9 & & 11 & 10 & & $86.3 \%$ & \\
\hline
\end{tabular}

before NT, or does LN with fibrosis really mean there is tumor cell ever? These issues should be research in the future study.

Although more studies, including randomized clinical trials are needed, our results indicate that the LRG system may be an independent predictive factor for distant metastasis and DFS of rectal cancer patients after NT and radical surgery.

\section{MATERIALS AND METHODS}

\section{Patients}

The study was approved by local ethic committees of all participating institutions.

We examined records of 347 patients with primary mid-rectal or distal rectal cancer who had received preoperative neoadjuvant therapy followed by radical surgery at four hospitals between June 2004 and October 2011. The study inclusion/exclusion criteria were: (1) rectal adenocarcinoma confirmed by pretreatment biopsy and/ or surgical resection with a total mesorectal excision; (2) locally advanced resectable disease (clinical stage II and III) with the distal margin located no farther than $10 \mathrm{~cm}$ from the anal verge; (3) no evidence of distant metastasis; and (4) patients underwent neoadjuvant therapy.

\section{Neoadjuvant therapy schedule}

Because there is currently no international consensus with regard to the indications for neoadjuvant chemoradiation therapy, patients managed with preoperative radiochemotherapy or preoperative radiotherapy were identified in our retrospective study. All patients received preoperative radiotherapy (50 Gy/2 Gy/25 f). Among those, 213 (61.4\%) patients were concurrently treated with chemotherapy (capecitabine, 825 $\mathrm{mg} / \mathrm{m}^{2} / \mathrm{bid}$ ), and the rest received radiotherapy alone. All patients received the same capecitabine regimen $(1000 \mathrm{mg} /$ $\mathrm{m}^{2} /$ bid, d1-14, 4-6 cycles) 3 weeks after radical surgery, except $62(17.9 \%)$ patients who rejected chemotherapy due to their age, poor physical condition, or side effects.

\section{Pathologic examination}

All sections of resection specimens were examined specially by local pathologists blinded to patients' clinical outcomes according to a standardized protocol that included AJCC TNM category, stage grouping, numbers of examined 
and involved lymph nodes, presence or absence of lymphatic, venous invasion, tumor deposits, TRG and LRG. The lymphatic or venous invasion was identified by morphology using Hematoxylin-Eosin (HE) staining. TDs were assessed using the 3-mm (TNM5) and contour (TNM6) rules.

\section{Lymph nodes regression grading}

Pathologic evaluation of primary tumor regression was performed according to Dworak et al [18], by determining the amount of viable tumor versus fibrotic tissue, which ranged from the lack of tumor regression to complete response with no viable tumor detected. The three groups of TRG and LRG scores were as follows: score 0 , total regression (no viable tumor cells; fibrotic mass only); score 1, intermediate regression; score 2, minor regression (dominant tumor mass with obvious fibrosis $\leq 25 \%$ of tumor mass), and no regression. Nonmetastatic lymph nodes were distinguished from LRG 0 (pCR) by absence of fibrosis. Nodal metastasis regression was evaluated using the same parameters of tumor regression grading referring to each metastatic lymph node. When different LRG scores were identified in one patient, only the most severe score was considered. However, we checked the preoperative CT and MRI pictures and found some patients had LN+ lesions, while pathologists did not found any $\mathrm{LN}$ with residual tumor cells and fibrosis (ypN0). Based on that, we assumed that NT killed all the tumor cells but pathologists could not find the residual lymph nodes. This group of patients should have good tumor regression in lymph nodes; thus, these patients (31 cases; 15.6\%) were enrolled into LRG 0. Besides, we also found 43 patients $(12.4 \%)$ with ypN0 but with TRG 2. After evaluating CT and MRI images, the 43 patients were categorized as cN0 and enrolled in LRG 2.

\section{Follow-up}

The follow-up results were collected from all four hospitals databases. The end-point of the follow-up was March 2015. The median time of follow-up was 60 months (26-129 months).

\section{Statistical analysis}

Spearman correlation test was used to assess relationship between TRG and LRG. Local recurrence and distant metastasis analyses were performed for all eligible patients who received $\mathrm{R} 0$ resection without distant metastasis found at time of surgery after neoadjuvant therapy. All time-to-event end-points were measured from date of radical surgery. Disease-free survival (DFS) was calculated from radical resection to finding evidence of recurrence and/or distant metastasis. Statistical analysis was performed using SPSS software (version 18). Differences were evaluated with the log-rank test. Analyses for local recurrence and distant metastasis were calculated as cumulative incidences. Mutivariable models were performed using the Cox proportional hazards model. All significant variables in the univariable analysis were included in multivariable Cox regression models in a forward-step procedure. The variables were entered in the order according to clinical relevance into the regression models with increasing complexity, and significance was assessed using analysis of variance analysis. A two-sided $p$ value less than 0.05 was considered significant.

\section{CONFLICTS OF INTEREST}

The authors indicated no potential conflicts of interest.

\section{Author contributions}

All authors contributed to this work. JL, JTY and SSX conceived and designed the study. JL, JTY, HL, JY, JJH, CL, XKN and BL provided study materials and analyzed the patient data. JL, SL and FD prepared figures and tables. JL, SSX and JTY wrote the manuscript. All authors reviewed the manuscript.

\section{Editorial note}

This paper has been accepted based in part on peer-review conducted by another journal and the authors' response and revisions as well as expedited peer-review in Oncotarget.

\section{REFERENCES}

1. Sauer R, Becker H, Hohenberger W, Rödel C, Wittekind C, Fietkau R, Martus P, Tschmelitsch J, Hager E, Hess CF, Karstens JH, Liersch T, Schmidberger H, Raab R; German Rectal Cancer Study Group. Preoperative versus postoperative chemoradio- therapy for rectal cancer. N Engl J Med. 2004; 351: 1731-40.

2. Collette L, Bosset JF, den Dulk M, Nguyen F, Mineur L, Maingon P, Radosevic-Jelic L, Piérart M, Calais G;European Organisation for Research and Treatment of Cancer Radiation Oncology Group. Patients with curative resection of cT3-4 rectal cancer after preoperative radiotherapy or radiochemotherapy: does anybody benefit from adjuvant fluorouracil-based chemotherapy? J Clin Oncol. 2007; 25: 4379-86.

3. Chetty R, Gill P, Bateman AC, Driman DK, Govender D, Bateman AR, Chua YJ, Greywoode G, Hemmings C, Imat I, Jaynes E, Lee CS, Locketz M, Rowsell C, Rullier A, Serra S, Szentgyorgyi E, Vajpeyi R, Delaney D, Wang LM. Pathological grading of regression: An International Study Group perspective. J Clin Pathol. 2012; 65: 865-866.

4. Perez RO, Habr-Gama A, Nishida Arazawa ST, Rawet V, Coelho Siqueira SA, Kiss DR, Gama-Rodrigues JJ. Lymph node micrometastasis in stage II distal rectal cancer following neoadjuvant chemoradiation therapy. Int $\mathrm{J}$ Colorectal Dis. 2005; 20: 434-9. 
5. Hughes R, Glynne-Jones R, Grainger J, Richman P, Makris A, Harrison M, Ashford R, Harrison RA,Livingstone JI, McDonald PJ, Meyrick Thomas J, Mitchell IC, Northover JM, Phillips R, Wallace M, Windsor A, Novell JR. Can pathological complete response in the primary tumour following preoperative pelvic chemoradiotherapy for T3-T4 rectal cancer predict for sterilisation of pelvic lymph nodes, a low risk of local recurrence and the appropriateness of local excision? Int J Colorectal Dis. 2006; 21: 11-7.

6. Read TE, Andujar JE, Caushaj PF, Johnston DR, Dietz DW, Myerson RJ, Fleshman JW, Birnbaum EH,Mutch MG, Kodner IJ. Neoadjuvant therapy for rectal cancer: histologic response of the primary tumor predicts nodal status. Dis Colon Rectum. 2004; 47: 825-31.

7. Caricato M, Ausania F, De Dominicis E, Vincenzi B, Rabitti C, Tonini G, Cellini F, Coppola R. Tumor regression in mesorectal lymph nodes after neoadjuvant chemoradiation for rectal cancer. EJSO. 2007; 33: 724-728.

8. Salzer-Kuntschik M, Delling G, Beron G, Sigmund R. Morphological grades of regression in osteosarcoma after polychemotherapy-study COSS 80. J Cancer Res Clin Oncol. 1983; 106: 21-4.

9. Bouzourene H, Bosman FT, Seelentag W, Matter M, Coucke P. Importance of tumor regression assessment in predicting the outcome in patients with locally advanced rectal carcinoma who are treated with preoperative radiotherapy. Cancer Feb. 2002; 94: 1121-30.

10. Mandard AM, Dalibard F, Mandard JC, Marnay J, Henry-Amar M, Petiot JF, Roussel A, Jacob JH, Segol P,Samama G,. Pathologic assessment of tumor regression after preoperative chemoradiotherapy of esophageal carcinoma. Clinicopathologic correlations. Cancer. 1994; 73: 2680-6.

11. Rödel C, Martus P, Papadoupolos T, Füzesi L, Klimpfinger M, Fietkau R, Liersch T, Hohenberger W, Raab R, Sauer
R, Wittekind C. Prognostic significance of tumor regression after preoperative chemoradiotherapy for rectal cancer. J Clin Oncol. 2005; 23: 8688-8696.

12. Fokas E, Liersch T, Fietkau R, Hohenberger W, Beissbarth T, Hess C, Becker H, Ghadimi M, Mrak K, Merkel S, Raab HR, Sauer R, Wittekind C2, Rödel C. Tumor regression grading after preoperative chemoradiotherapy for locally advanced rectal carcinoma revisited: updated results of the $\mathrm{CAO} / \mathrm{ARO} /$ AIO-94 trial. J Clin Oncol. 2014; 32: 1554-1562.

13. Kalady MF, de Campos-Lobato LF, Stocchi L, Geisler DP, Dietz D, Lavery IC, Fazio VW. Predictive factors of pathologic complete response after neoadjuvant chemoradiation for rectal cancer. Ann Surg. 2009; 250: 582-589.

14. Habr-Gama A, Perez RO, Nadalin W, Sabbaga J, Ribeiro U Jr, Silva e Sousa AH Jr, Campos FG, Kiss DR, GamaRodrigues J. Operative versus nonoperative treatment for stage 0 distal rectal cancer following chemoradiation therapy: long-term results. Ann Surg. 2014; 240: 711-717.

15. Maas M, Beets-Tan RG, Lambregts DM, Lammering G, Nelemans PJ, Engelen SM, van Dam RM,Jansen RL, Sosef M, Leijtens JW, Hulsewé KW, Buijsen J, Beets GL. Wait-and-see policy for clinical complete responders after chemoradiation for rectal cancer. J Clin Oncol. 2011; 29: 4633-4640.

16. Li J, Liu H, Yin J, Liu S, Hu J, Du F, Yuan J, Lv B, Fan J, Leng S, Zhang X. Wait-and-see or radical surgery for rectal cancer patients with a clinical complete response after neoadjuvant chemoradiotherapy: a cohort study. Oncotarget. 2015; 6: 42354-61. doi: 10.18632/oncotarget.6093.

17. Li J, Liu H, Hu J, Liu S, Yin J, Du F, Yuan J, Lv B. New tumor regression grade for rectal cancer after neoadjuvant therapy and radical surgery. Oncotarget. 2015; 6: 42222-31. doi: 10.18632/oncotarget.6008.

18. Dworak O, Keilholz L, Hoffmann A. Pathological features of rectal cancer after preoperative radiochemotherapy. Int J Colorectal Dis. 1997; 12: 19-23. 